\title{
pn-CCDs in a Low-Background Environment: Detector Background of the CAST X-ray Telescope
}

\author{
M. Kuster ${ }^{a, d}$, S. Cebrián ${ }^{b}$, A. Rodríquez ${ }^{b}$, R. Kotthaus ${ }^{c}$, H. Bräuninger $^{d}$, J. Franz ${ }^{e}$, P. Friedrich $^{d}$, \\ R. Hartmann ${ }^{f}$, D. Kang ${ }^{e}$, G. Lutz $^{c}$, L. Strüder ${ }^{d}$ \\ ${ }^{a}$ Technische Universität Darmstadt, Schlossgartenstr. 9, 64289 Darmstadt, Germany \\ ${ }^{b}$ Instituto de Física Nuclear y Altas Energías, Universidad de Zaragoza, Zaragoza, Spain \\ ${ }^{c}$ Max-Planck-Institut für Physik, Föhringer Ring 6, 80805 München, Germany \\ ${ }^{d}$ Max-Planck-Institut für extraterrestrische Physik, Giessenbachstr., 85748 Garching, Germany \\ ${ }^{e}$ Universität Freiburg - Physikalisches Institut, Herrman-Herder-Str. 3, 79104 Freiburg, Germany

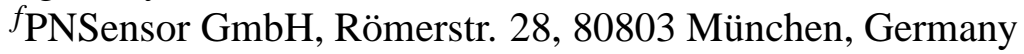

\begin{abstract}
The CAST experiment at CERN (European Organization of Nuclear Research) searches for axions from the sun. The axion is a pseudoscalar particle that was motivated by theory thirty years ago, with the intention to solve the strong CP problem. Together with the neutralino, the axion is one of the most promising dark matter candidates. The CAST experiment has been taking data during the last two years, setting an upper limit on the coupling of axions to photons more restrictive than from any other solar axion search in the mass range below $10^{-1} \mathrm{eV}$. In 2005 CAST will enter a new experimental phase extending the sensitivity of the experiment to higher axion masses.

The CAST experiment strongly profits from technology developed for high energy physics and for X-ray astronomy: A superconducting prototype LHC magnet is used to convert potential axions to detectable X-rays in the $1-10 \mathrm{keV}$ range via the inverse Primakoff effect. The most sensitive detector system of CAST is a spin-off from space technology, a Wolter I type X-ray optics in combination with a prototype pn-CCD developed for ESA's XMM-Newton mission. As in other rare event searches, background suppression and a thorough shielding concept is essential to improve the sensitivity of the experiment to the best possible. In this context CAST offers the opportunity to study the background of pn-CCDs and its long term behavior in a terrestrial environment with possible implications for future space applications. We will present a systematic study of the detector background of the pn-CCD of CAST based on the data acquired since 2002 including preliminary results of our background simulations.
\end{abstract}

Keywords: Solar Axions, Dark Matter, pn-CCD, X-ray Optics, CAST, Low Background

\section{INTRODUCTION}

The most sensitive axion helioscope in operation, the CERN axion solar telescope - CAST, aiming to discover the elusive particle axion, has recently finished its first data taking period. Although no signal over background could be detected by any of the detectors of CAST during the first period of operation, the results from data taken during 2003 allow to improve the existing upper limit on the coupling of axions to photons to $g_{a \gamma}<1.16 \times 10^{-10} \mathrm{GeV}^{-1}$ (for $m_{\mathrm{a}}<0.02 \mathrm{eV}$, see Fig. 1). ${ }^{1}$ The axion as a particle is a direct consequence ${ }^{2,3}$ of the Peccei-Quinn mechanism ${ }^{4}$ proposed in 1977 to solve the still existing strong $\mathrm{CP}$ problem. The strong $\mathrm{CP}$ problem describes the fact that $\mathrm{CP}$ violation in strong interactions seems not to be realized in nature, albeit the QCD Lagrangian density contains CP violating terms. This inconsistency between theory and experiment becomes apparent by the fact, that the best experimental limit for the electric dipole moment of the neutron corresponds to $\approx 10^{-9}$ of the range allowed within QCD. As pointed out by several authors, e.g. G. Raffelt, ${ }^{5}$ axions of a mass in the sub $\mathrm{eV}$ range would also be a viable hot and cold dark matter candidate.

If the axion exists, it would couple to two photons with the strength given by the coupling constant $g_{a \gamma}$. This coupling would allow the production of axions inside the hot plasma of stars via the so called Primakoff effect $\left(\gamma \gamma^{\prime} \rightarrow\right.$ a), where photons interact with the coulomb field of plasma particles and are converted into axions. The expected axion flux emitted

Send correspondence to M. Kuster: E-mail: kuster@hll.mpg.de, Phone: +49 (0)6151 16-2321 


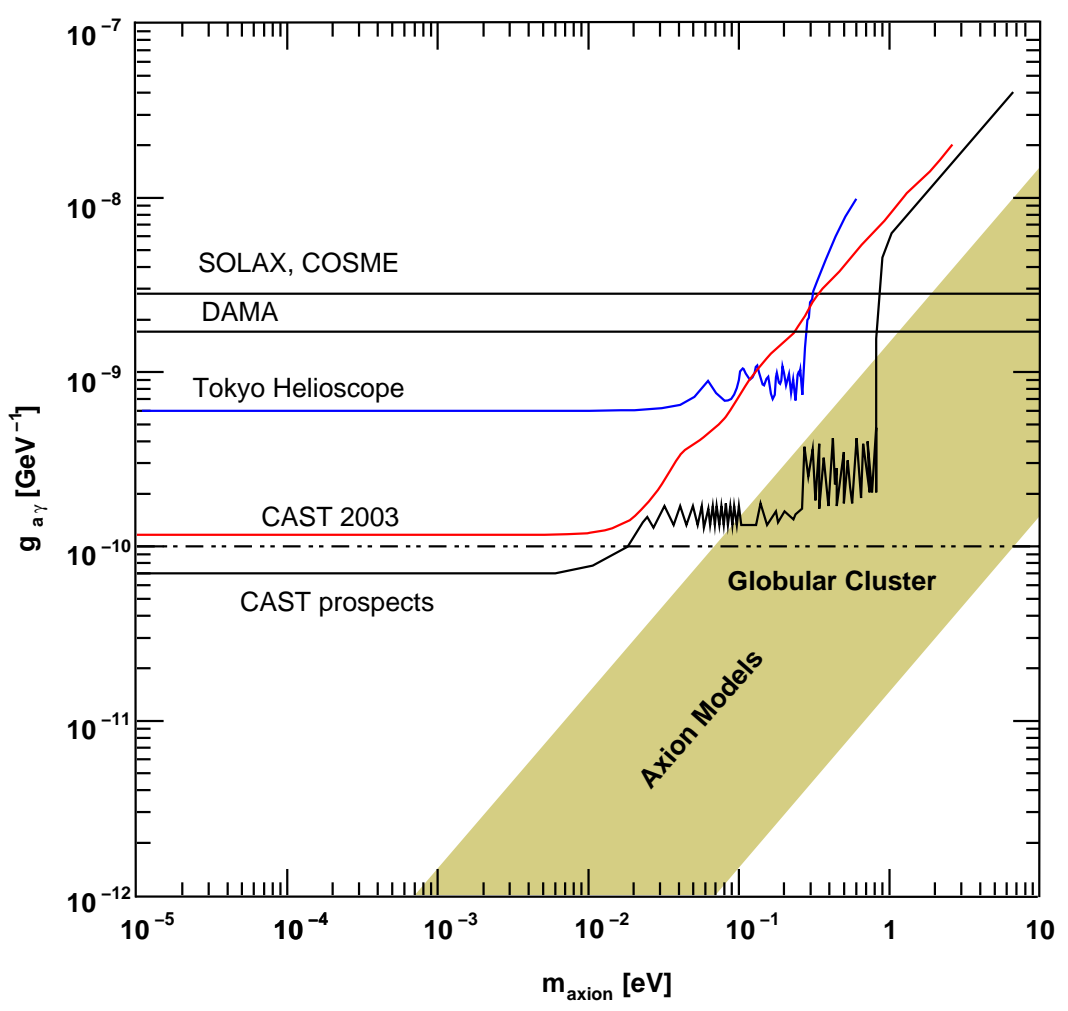

Figure 1. Axion mass $m_{\mathrm{a}}$ versus the axion to photon coupling constant $g_{a \gamma}$ parameter space. The red line marks the upper limit on $g_{a \gamma}$ derived from CAST data taken in 2003. The shaded area labeled "Axion Models" marks the region favored by theoretical axion models. Results from the previous experiments SOLAX, COSME, DAMA, and the Tokyo Helioscope are shown for comparison. The dash dotted line labeled "Globular Clusters" marks the limit on $g_{a \gamma}$ derived from astrophysical considerations. ${ }^{1}$

by the sun is $\Phi_{\mathrm{a}}=g_{10}^{2} \cdot 3.77 \times 10^{11}$ axions $\mathrm{cm}^{-2} \mathrm{sec}^{-1}$ corresponding to an axion luminosity of $L_{\mathrm{a}}=g_{10}^{2} \cdot 1.7 \times 10^{-3} L_{\odot}$ with $g_{10}=g_{a \gamma} \times 10^{10} \mathrm{GeV} .{ }^{5}$ The energy spectrum of the axions would reflect the thermal energy spectrum of the photons in the core of the sun with a mean energy of $\approx 4.2 \mathrm{keV}$. To detect axions on Earth, Sikivie ${ }^{6}$ proposed an experimental approach called the "axion helioscope" which the CAST experiment is based on: in the presence of a transverse magnetic field solar axions could be converted back to observable X-rays via the time reversed Primakoff effect. In CAST we use a 9 Tesla superconducting dipole magnet for this purpose, providing a homogeneous transversal magnetic field inside two $9.26 \mathrm{~m}$ long magnet tubes with a diameter of $43 \mathrm{~mm}$ each. The magnet is supported by a movable platform that allows to point the magnet to the sun for $3 \mathrm{~h}$ per day ( $1.5 \mathrm{~h}$ during sun rise and sun set). At each end of the magnet three $\mathrm{X}$-ray detectors (a TPC covering two magnet bores, a Micromegas detector, and an X-ray telescope) are looking for an excess signal over background in order to detect a possible axion signal. The probability for an axion to be converted to an X-ray photon in the magnetic field depends on the magnetic field strength $B$, the length of the magnetic field $L$, the momentum transfer from the axion to the photon $|\vec{q}|=\left|\frac{m_{a}^{2}-m_{\gamma}^{2}}{2 E}\right|$, the absorption coefficient $\Gamma$ of the medium inside the conversion volume, and the axion to photon coupling constant $g_{a \gamma}$ according to ${ }^{7}$ :

$$
P_{a \rightarrow \gamma}=\left(\frac{B g_{a \gamma}}{2}\right)^{2} \frac{1}{q^{2}+\Gamma^{2} / 4}\left[1+e^{-\Gamma L}-2 e^{-\Gamma L / 2} \cos (q L)\right]
$$

During the first phase of operation of CAST in 2003-2004 (Phase I) the conversion volume of the magnet was evacuated (i.e. $\Gamma=0$ and $m_{\gamma}=0$ ). Then the conversion probability depending on the axion mass remains constant for a given $g_{a \gamma}$ and given magnet parameters $B$ and $L$, as long as the momentum transfer from the axion to the outgoing photon is negligible or in other words $q L \ll \pi$. The expected integrated $\mathrm{X}$-ray photon flux in the energy range of $1-7 \mathrm{keV}$ is then $\Phi_{\gamma}=0.51 g_{10}^{4}\left(\frac{L}{9.26 \mathrm{~m}}\right)^{2}\left(\frac{B}{9 \mathrm{~T}}\right)^{2}$ photons $\mathrm{cm}^{-2} \mathrm{~d}^{-1}$. For higher axion masses, $m_{\mathrm{a}} \gtrsim 0.02 \mathrm{eV}$, the conversion probability 
rapidly drops and limits the sensitivity of the experiment with the magnet pipes being evacuated. For the end of 2005 it is planned to fill the magnet bore with a refractive medium $\left({ }^{4} \mathrm{He}\right.$ first and then $\left.{ }^{3} \mathrm{He}\right)$, such that the photon acquires an "effective" mass and the axion to photon momentum mismatch can be overcome (CAST Phase II). During Phase II, CAST has the potential to probe regions in the $g_{a \gamma}-m_{\mathrm{a}}$ parameter space that were not reachable for any other experiment so far. Especially the parameter range that is favored by theoretical axion models (region labeled "axion models" in Fig. 1) can experimentally be probed for the first time with CAST.

In 2003 the three detector systems of CAST have taken useful data for more than $260 \mathrm{~h}$ during axion sensitive conditions. In addition, more than $1233.5 \mathrm{~h}$ of detector background data were acquired in 2003 with the X-ray telescope under different operating conditions. This data base, plus $179.4 \mathrm{~h}$ of tracking data and $1723.5 \mathrm{~h}$ of background data from the data taking runs in 2004, allow a systematic study of the performance and of the background observed with the most sensitive detector system of CAST, the X-ray telescope in conjunction with the pn-CCD detector.

\section{THE CAST X-RAY TELESCOPE AND PN-CCD DETECTOR}

Since the axion to photon conversion inside the magnet tubes conserves the momentum of the incoming axion, the resulting $\mathrm{X}$-rays would leave the magnet bore as a nearly parallel beam. The divergence is given by the angular size of the magnet aperture fully covering the axion producing region of the sun. The X-ray flux can then either be observed directly with a detector mounted to the magnet bore, like it is the case for the Micromegas and TPC detectors, or it can be focused with an X-ray optics onto a focal plane detector with a high spatial resolution. The advantage of the latter approach is twofold: in case of a positive signal a telescope system would allow to aquire an image of the axion distribution in the core of the sun and the focusing of X-rays coming from the magnet bore from an area of $14.5 \mathrm{~cm}^{2}$ to a small spot with an area of $\approx 6.4 \mathrm{~mm}^{2}$ improves the signal to background ratio and thus the sensitivity of the experiment, significantly. In CAST we realized such an imaging system, consisting of a prototype Wolter I type X-ray telescope developed for the German X-ray satellite ABRIXAS ${ }^{8,9}$ and a pn-CCD detector similar to the fully depleted EPIC-pn focal plane detector of XMM-Newton. ${ }^{10,11}$ A detailed summary on the performance and characteristics of the pn-CCD detector of XMMNewton can be found in Ref. ${ }^{12}$ and references therein. The backside illuminated CCD chip of CAST is operated at a temperature stabilized at $-130^{\circ} \mathrm{C}$ and has a sensitive area of $2.88 \mathrm{~cm}^{2}$ divided into $200 \times 64$ square pixels, providing a quantum efficiency close to unity between $1-7 \mathrm{keV}$.

Fig. 2 is a picture showing the interior of the detector, the CCD chip, the cooling mask, and the electronic components necessary to operate the CCD. In addition the different detector components are shown as schematic drawings as represented in the GEANT4 model we use for background simulations. An electronics board is fixed to the rear side of the CCD, carrying electronic components soldered to the board. The CCD chip itself is glued onto an aluminum oxide substrate which is sandwiched by a Cu cooling mask from the front and the back, providing a good thermal coupling to the cold finger. The CCD chip is surrounded by a passive shield inside the vacuum vessel build of a 10-40 mm thick copper box made of low activity, oxygen free copper. The copper box is surrounded by a $22 \mathrm{~mm}$ thick lead layer free of ${ }^{210} \mathrm{~Pb}$, followed by additional $2.5 \mathrm{~cm}$ of lead outside the vacuum vessel. The detector in this final configuration was installed at the CAST magnet in spring 2004 before the 2004 data taking runs.

\subsection{Detector Performance}

The pn-CCD detector was operated almost continuously during the data taking periods of CAST in 2003 and 2004. In total we accumulated more than $2960 \mathrm{~h}$ of useful background data during both years, including daily calibration measurements with an ${ }^{55} \mathrm{Fe}$ source using a flat field illumination. This data set allows us to study parameters defining the performance of the detector in a low background environment over a period of 2 years. The most important detector parameters, the detector noise averaged over all pixels, the charge transfer inefficiency (CTI), the amplification (i.e. the ADU to keV conversion), and the dark current (offset) averaged over all pixels are summarized in Fig. 3 for the data taking period of 2004. Similar results could be derived for the 2003 data. All parameters except the energy resolution given in FWHM of the $\mathrm{Mn}-\mathrm{K}_{\alpha}$ line and the detector noise show no significant variation over the entire period of operation. The increase of detector noise (mid June and at the end of October) is correlated to an overall increase of noise in the experimental area of CAST. This change of noise is apparent from the width of the $\mathrm{Mn}-\mathrm{K}_{\alpha}$ given as FWHM as well. A degradation of, e.g. the CTI due to radiation damage defects like it is the case for the CCD detectors of XMM-Newton operated in orbit, is not expected in a ground based environment. 


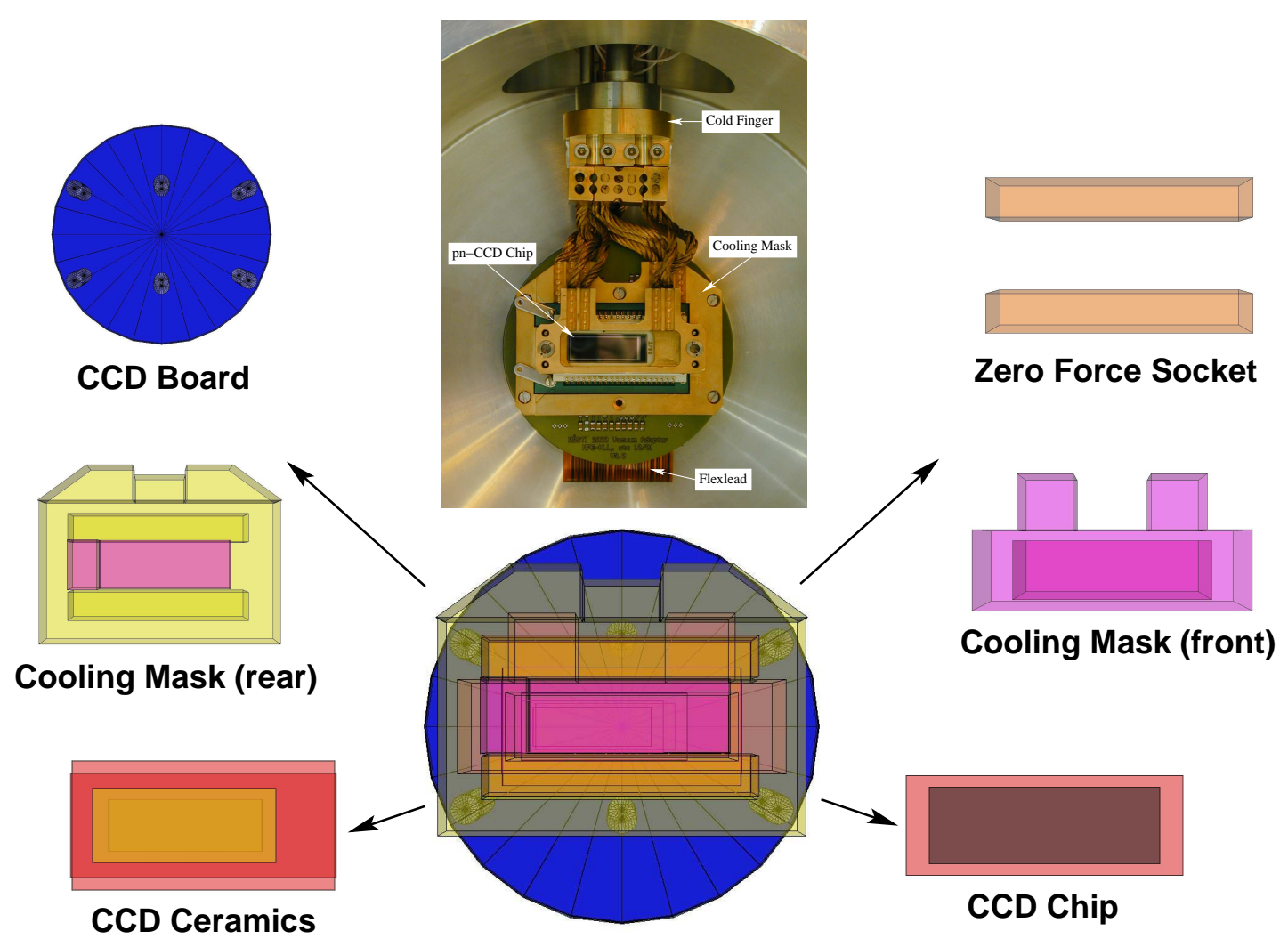

Figure 2. Top Center: The pn-CCD chip (black part in the center) with the gold plated cooling mask which is connected to a cold finger of a Stirling cooler device. The individual detector components as modeled in GEANT4 are shown below and to the left and right. Due to the limited time for R\&D these components were tested for their natural radioactivity in the Canafranc underground laboratory, ${ }^{10}$ but not built from selected and radio-pure materials.

\section{THE DETECTOR BACKGROUND}

The main contributions to the overall background of the CAST pn-CCD detector is external background induced by cosmic rays, gamma rays, and radioactive impurities of structural magnet materials. In addition the intrinsic detector background due radioactive impurities in the detector materials, or the shape of the response function of the pn-CCD can significantly contribute to the total observed background.

\subsection{Spectral Distribution}

A time averaged background spectrum observed in 2004 under the same operating conditions as during sun observations is shown in Fig. 4. The axion signal is expected as an excess signal in the energy band between 1 and $7 \mathrm{keV}$ where the background spectrum has its minimum. The background level in this energy range corresponds to a mean differential flux of $(7.69 \pm 0.07) \times 10^{-5}$ counts $\mathrm{cm}^{-2} \mathrm{sec}^{-1} \mathrm{keV}^{-1}$ which is equivalent to an integral background count rate of $0.1 \mathrm{counts}^{-1}$ in the focal spot with an area of $6.4 \mathrm{~mm}^{2}$. The features dominating the background spectrum are fluorescent emission lines from $\mathrm{Au}, \mathrm{Cu}$, and $\mathrm{Pb}$ originating in the materials close to the $\mathrm{CCD}$ chip on top of a Compton like continuum spectrum with a slightly negative slope. The $\mathrm{Pb}$ lines originate presumably form the "ordinary solder" that was used to fabricate the electronics board. This solder can be contaminated by e.g. ${ }^{210} \mathrm{~Pb}$. At energies below $1 \mathrm{keV}$ a broad noise peak dominates the spectrum which is observed in the CERN environment only. This background contribution seems to be caused by the unusual high electronic noise level in the CAST area compared to controlled laboratory conditions. Since the building where the CAST experimental area is located, also serves as an electronic support point for the Large Hadron ColliderLHC and in general was not foreseen and designed as an experimental area from the beginning, the electronic noise level 


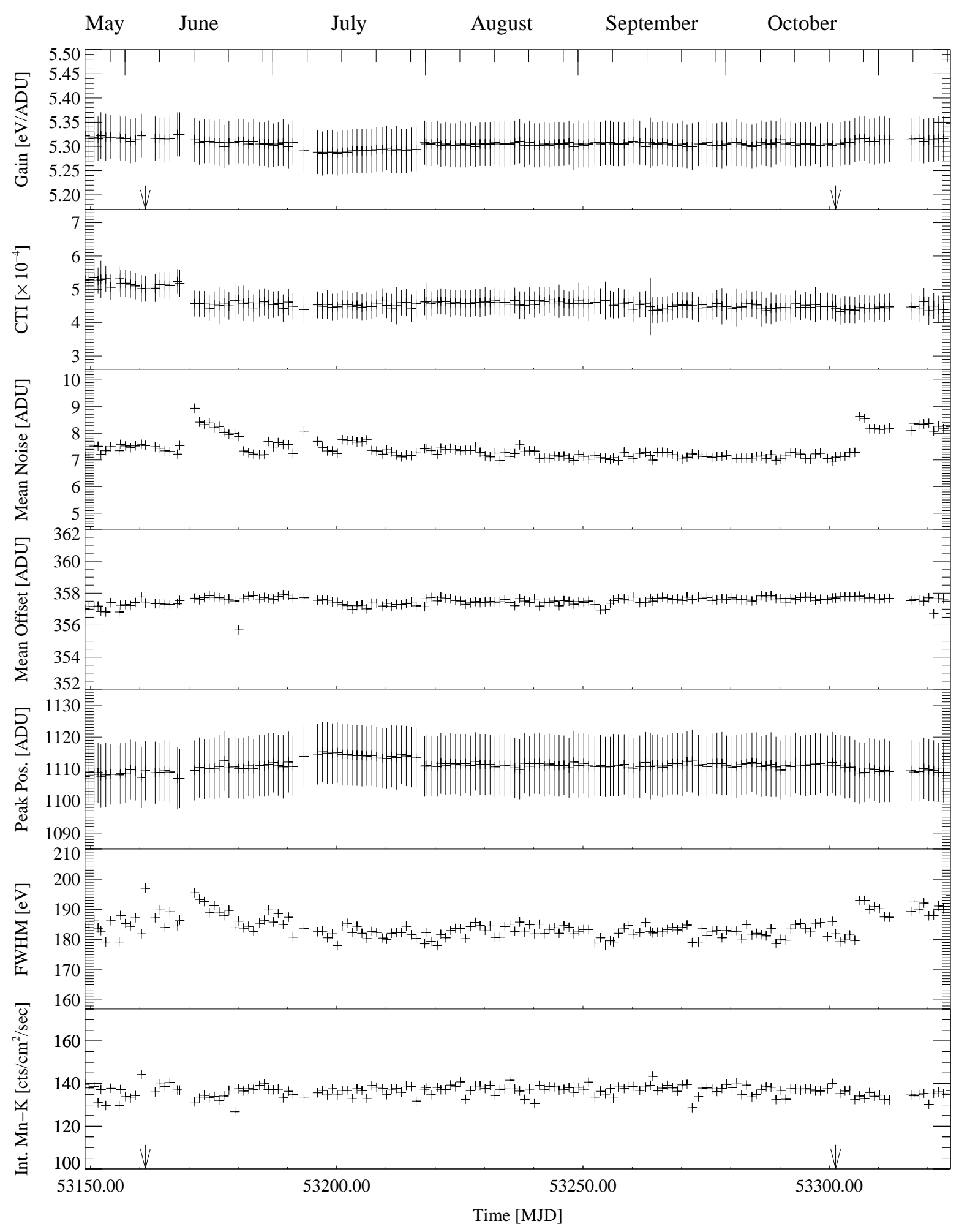

Figure 3. Performance of the pn-CCD Detector of CAST during the data taking period of 2004. From top to bottom: The amplification (ADU to keV conversion), charge transfer inefficiency (CTI), mean noise averaged over all pixels, the mean offset averaged over all pixels, the peak position of the $\mathrm{Mn}-\mathrm{K}_{\alpha}$ line of the calibration source, the energy resolution, and the intensity of the Mn- $\mathrm{K}_{\alpha}$ are shown. None of the detector parameters shows a significant variation over the time of operation. 


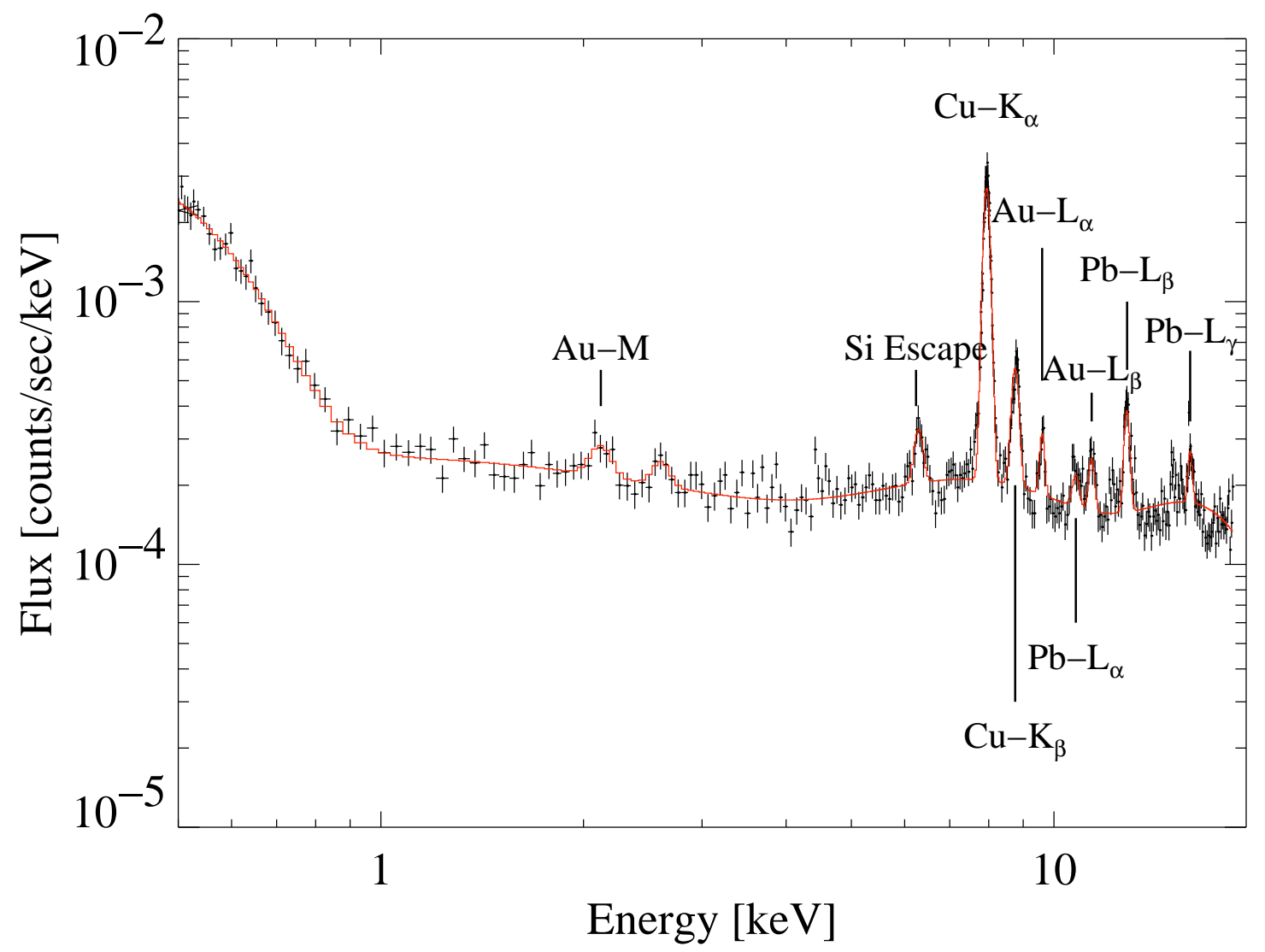

Figure 4. Background spectrum observed with the pn-CCD detector in the CAST environment. The overall background is composed of contributions from fluorescent emission of $\mathrm{Pb}, \mathrm{Cu}$, and $\mathrm{Au}$ on top of a continuum spectrum. The peak at energies $E<1 \mathrm{keV}$ corresponds to low energy noise. The X-rays from axion to photon conversion are expected to be thermally distributed between $1.0 \mathrm{keV}$ and $8 \mathrm{keV}$.
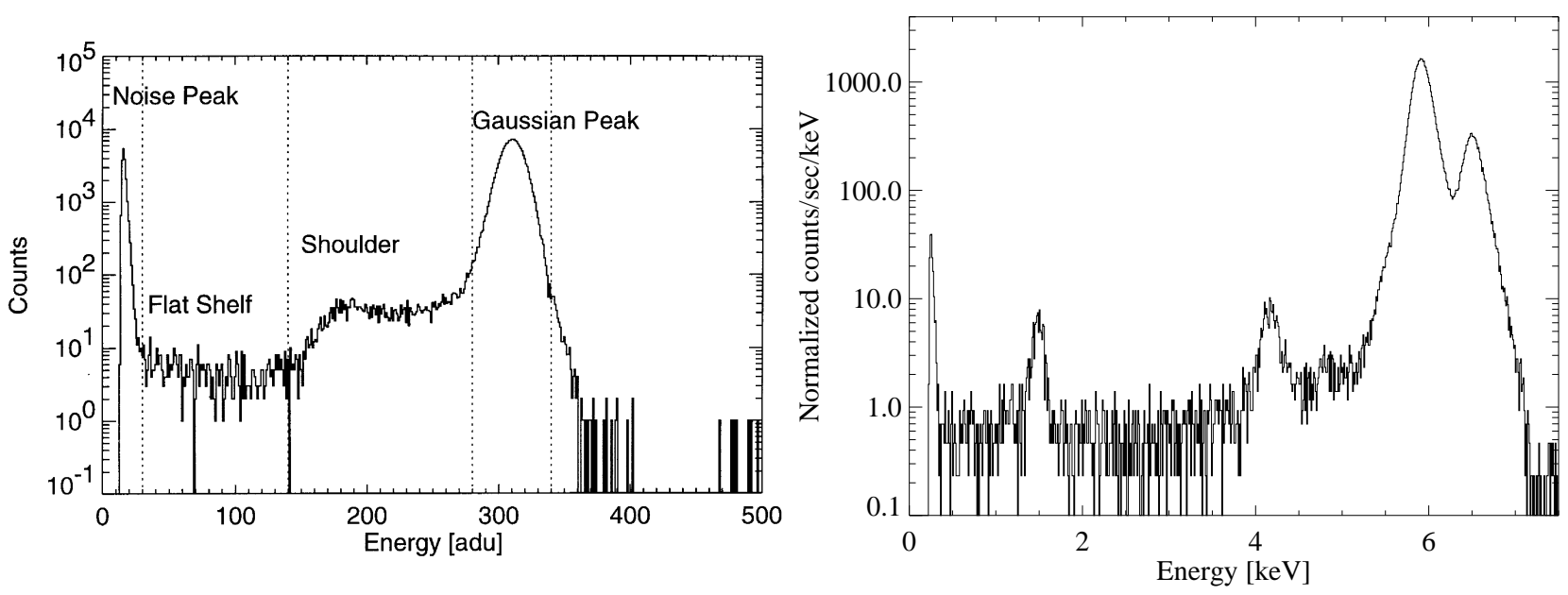

Figure 5. Left: Spectral energy response of an XMM-Newton EPIC pn-CCD similar to the one in use for CAST, for an incident monochromatic photon energy of $1.4 \mathrm{keV} .{ }^{13}$ Right: Spectrum of an ${ }^{55}$ Fe calibration source measured with the CAST detector. The Al-K line apparent at $1.5 \mathrm{keV}$ originates from an aluminum filter installed in front of the ${ }^{55} \mathrm{Fe}$ calibration source. 


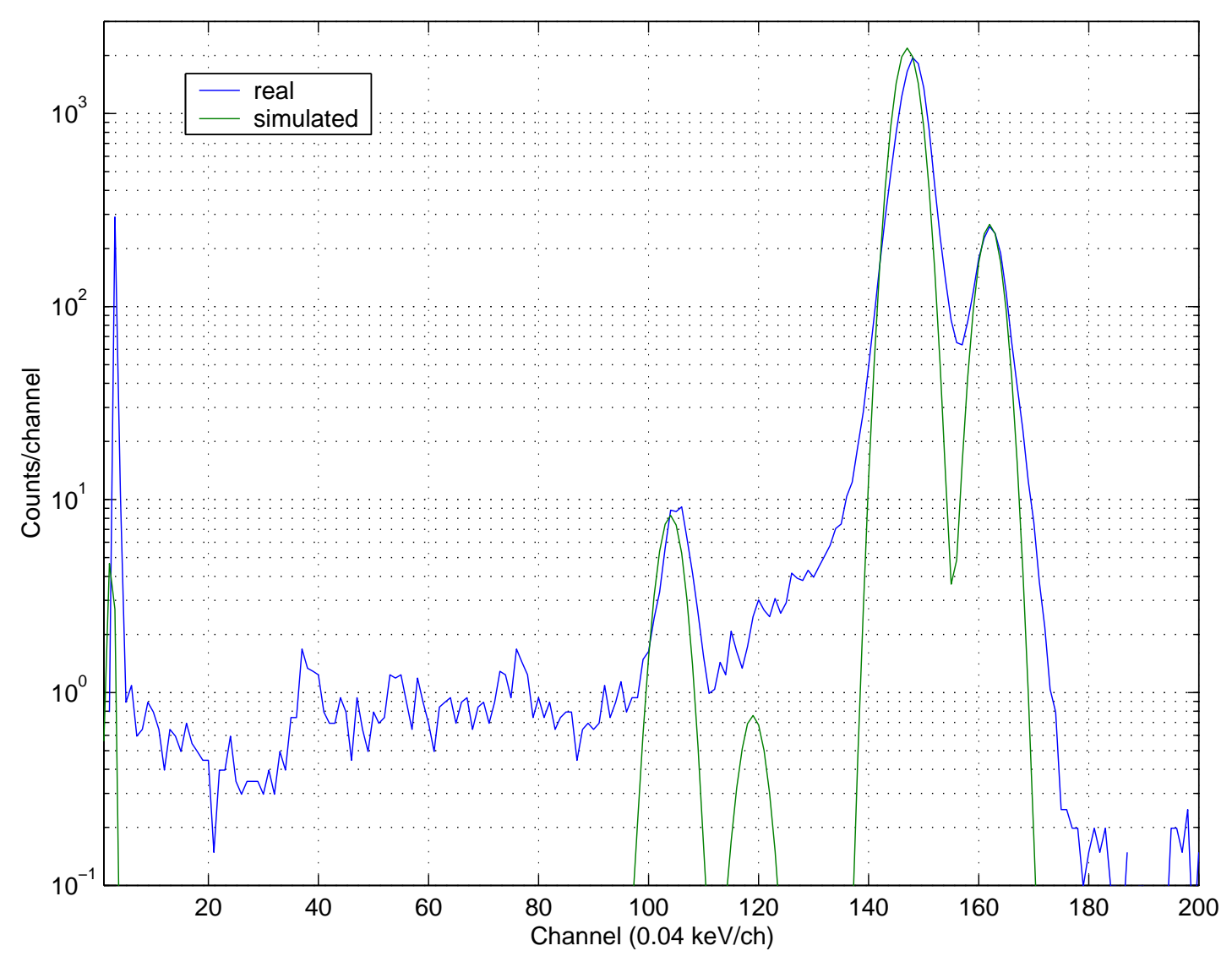

Figure 6. A simulated ${ }^{55} \mathrm{Fe}$ calibration spectrum (green line) in comparison to a measured spectrum (blue line). The underground apparent between channel 10 and 140 is due to non-linearities in the detector response function (see Fig. 5).

is unusual high and variable with time. This low energy background component is limited to energies $E<0.8 \mathrm{keV}$, the region outside the axion sensitive energy range, and it does not effect the sensitivity of CAST.

\subsection{Influence of the Detector Response Function}

Due to secondary energy loss effects in the semiconductor (partial-event effect ${ }^{13}$ ), the response of the pn-CCD detector to mono-energetic photons is not a mere Gaussian, as expected from an ideal detector with a finite energy resolution, but is slightly distorted for incident photon energies with $E<6 \mathrm{keV}$. As an example, a spectrum of monoenergetic X-rays with an energy of $1.4 \mathrm{keV}$ measured with the pn-CCD detector is shown in Fig. 5. It is apparent, that the Gaussian shaped main photo-peak is asymmetrically distorted towards lower energies ("Shoulder") and additional photons are detected equally distributed between the noise peak and the shoulder of the Gaussian main peak ("flat shelf"). The shape, height, and the width of the shoulder as well as the flux level of the flat shelf have been determined during the ground calibration campaign of the pn-CCD detector of XMM-Newton. We refer the interested reader to Ref. ${ }^{13,14}$, for a more detailed introduction to the partial-event effect and its implications on the spectral response of a pn-CCD. The parameters that define the shape of the shoulder and the flat shelf generally are energy dependant. Both effects, the asymmetric shoulder and the flat shelf, are most distinct for incident photon energies $E<6 \mathrm{keV} .{ }^{14}$ Due to this redistribution effect especially photons in the flat shelf could contribute to the overall background, if emission lines are present in the background spectrum. For the CAST pn-CCD the ratio between hight of the Gaussian peak and the level of the flat shelf (peak to valley ratio) is approximately 2700 for the $\mathrm{Mn}-\mathrm{K} \alpha$ line as shown in the right part of Fig. 5. Taking this ratio into account the contribution of redistribution effects to the low energy background would be two orders of magnitude below the actual level of sensitivity and therefore is negligible. 


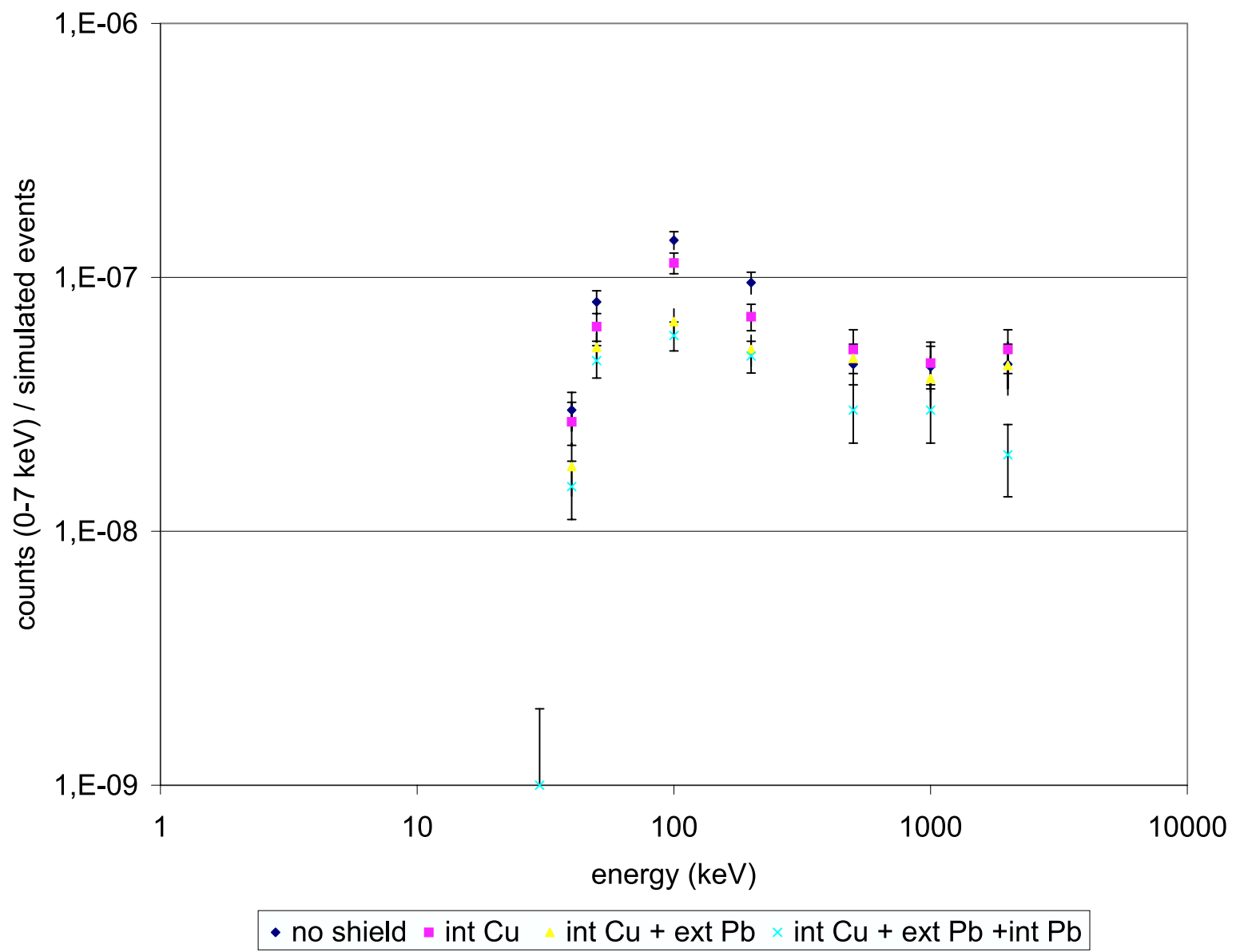

Figure 7. Response of the pn-CCD to external $\gamma$-ray background. The energy of the incident $\gamma$-ray photon versus the number of detected counts in the $0-7 \mathrm{keV}$ is shown relative to the number of simulated events for different configurations of the shield. Results are shown for an unshielded detector, the copper box (int $\mathrm{Cu}$ ), an additional $\mathrm{Pb}$ shield outside the vessel (ext $\mathrm{Pb}$ ), and an additional $\mathrm{Pb}$ shield inside the vessel being installed (int $\mathrm{Pb}$ ).

\section{BACKGROUND SIMULATIONS}

To understand the origin of the background observed with the CAST pn-CCD and to quantify the relative contributions of different sources of background we made extensive Monte-Carlo simulations with the GEANT4 package. As a first step, a calibration spectrum and the quantum efficiency of the CCD were simulated to verify the reliability of the low energy physical models implemented in GEANT4 and our simulation code. In general, the response on a ${ }^{55} \mathrm{Fe}$ source is very well reproduced including the area ratio of the Si escape peak to the main emission peak (see Fig. 6). However, the simulated quantum efficiency in the $10-15 \mathrm{keV}$ energy range is slightly higher, than the results from calibration measurements.

\subsection{External $\gamma$-ray Background}

The energy dependant response of the pn-CCD to $\gamma$ 's with an energy $10 \mathrm{keV}<E<2 \mathrm{MeV}$ has been studied. For the simulations we assumed a simplified spherical geometry with isotropic emission for the incident external $\gamma$-ray background, and subsequently different configurations of the pn-CCD passive shield have been taken into consideration. The integral number of detected counts in the energy range $0-7 \mathrm{keV}$ originating from high energy $\gamma$ 's is shown in Fig. 7. From our results it is obvious that $\gamma$-rays with an energy close to $100 \mathrm{keV}$ contribute most to the pn-CCD background and that the energy dependence of the contribution of the external $\gamma$-ray background is low. The Pb shield suppresses mainly $\gamma$ 's with an energy above $100 \mathrm{keV}$, while the copper shield has only a marginal effect on the $\gamma$ background. From the simulation 


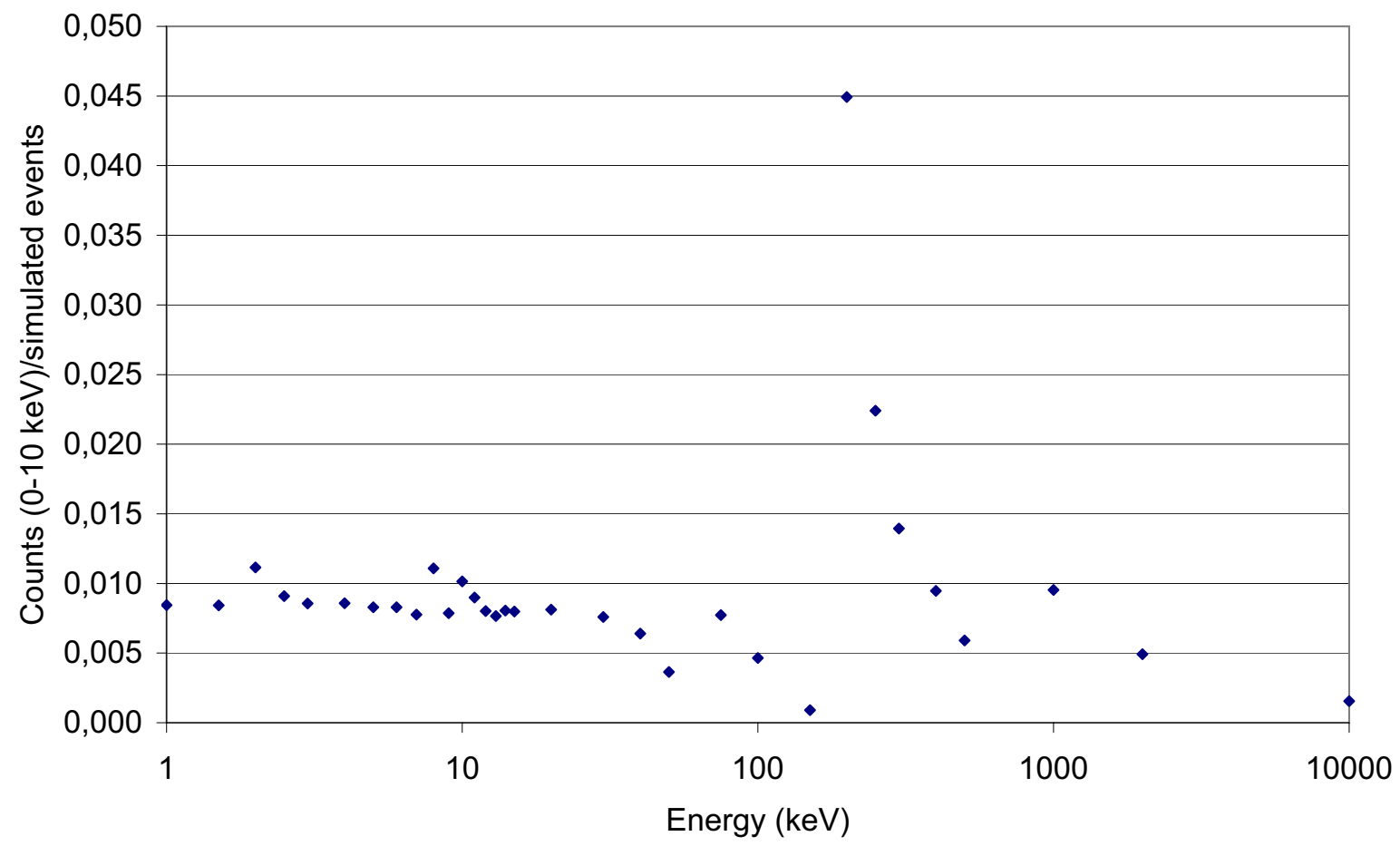

Figure 8. Response of the pn-CCD to the neutron background.

we can derive a relative background reduction of a factor of 1.6-2.5 between an unshielded detector and the final shield configuration of the CAST pn-CCD detector, which includes the internal $\mathrm{Cu}$ box, the internal $\mathrm{Pb}$ shield, and the external $\mathrm{Pb}$ shield. The simulations underestimate the background reduction we have derived from background measurements under different shielding configurations, which is a factor of 3 between an unshielded detector and the detector with its full shield being installed. The reason of this discrepancy might be the simplifications in the detector geometry we made, the uncertainties in the response of the pn-CCD to high energy $\gamma$ 's, and/or the isotropic $\gamma$ emission geometry assumed for the simulations. In addition, the background of internal sources in the detector set-up (natural radioactivity) has been neglected in these simulations.

\subsection{External Neutron Background}

Similar to the study for the $\gamma$-ray background, the influence of thermal and high energy neutrons on the total background of the pn-CCD has been evaluated. The energy of the incident neutrons has been varied from $10^{-2} \mathrm{eV}$ up to $10 \mathrm{MeV}$. In Fig. 8 the ratio between events detected in the $0-10 \mathrm{keV}$ energy range of visible energy (taking the quenching factor into account) and the total number of simulated events as a function of the incident energy of the neutron is shown. The process that dominates the interaction of neutrons with the detector material seem to be elastic scattering of neutrons off the silicon nuclei. The resonance visible in Fig. 8 corresponds to a resonance in the neutron elastic scattering cross-section for silicon. Taking into account the typical neutron flux at sea level ${ }^{15,16}$ the differential count rate expected in the pn-CCD detector from high energy neutron interactions can be estimated to $\approx 6 \times 10^{-6}$ counts $\mathrm{cm}^{-2} \mathrm{sec}^{-1} \mathrm{keV}^{-1}$, which is below the actual sensitivity of the detector. Although the spectral distribution of the cosmic-ray induced neutron flux has a maximum at thermal energies the estimated differential count rate of $3 \times 10^{-8}$ counts $\mathrm{cm}^{-2} \mathrm{~s}^{-1} \mathrm{keV}^{-1}$ originating in thermal neutron interactions is two orders of magnitude smaller compared to the rates expected from high energy neutrons. Finally we roughly estimated the muon-induced neutron background, which is around two orders of magnitude lower than the flux of environmental neutrons. 


\section{CONCLUSIONS AND PROSPECTS}

We have presented preliminary results from our study of the background of the pn-CCD detector of CAST. Taking into account the results of our simulations and background measurements we can conclude that effects due to the non-linear detector response of the pn-CCD and neutron induced background are negligible and do not contribute to the background of the CAST pn-CCD detector at the present level of sensitivity. The major contributions to the background seem to be environmental $\gamma$ background, internal natural radioactivity of the detector materials, and muon induced background. In order to quantify these contributions, further simulations based on a refined detector geometry are in progress. Absolute estimates of the background and the spectral shape of the background are difficult to obtain, especially when the incident spectrum of, e.g. environmental $\gamma$ 's is not known. Measurements on environmental background has been performed recently in the CAST experimental environment and will further help to improve the simulations and modeling of the background observed with the pn-CCD.

\section{ACKNOWLEDGMENTS}

We acknowledge support by the Bundesministerium für Bildung und Forschung (BMBF) under the grant number 05 CC2EEA/9, the N3 Dark Matter network of the Integrated Large Infrastructure for Astroparticle Science - ILIAS, and the Virtuelles Institut für Dunkle Materie und Neutrinos - VIDMAN. This work has been performed within the CAST collaboration, we thank our colleagues for their support.

\section{REFERENCES}

1. K. Zioutas, S. Andriamonje, V. Arosov, S. Aune, F. T. Aune, D. Autiero, F. Avignone, K. Barth, A. Belov, B. Beltran, H. Bräuninger, J. M. Carmona, S. Cebrian, E. Chesi, J. Collar, R. Creswick, T. Dafni, M. Davenport, L. DiLella, C. Eleftheriadis, J. Engelhauser, G. Fanourakis, H. Farach, E. Ferrer, H. Fischer, F. Formenti, J. Franz, T. Geralis, I. Giomataris, S. Gninenko, N. Golubev, M. Hasinoff, F.-H. Heinsius, D. H. H. Hoffmann, I. Irastorza, J. Jacoby, D. Kang, K. Königsmann, R. Kotthaus, M. Krcmar, K. Kousouris, M. Kuster, B. Lakic, C. Lasseur, A. Liolios, A. Ljubicic, G. Lutz, G. Luzon, D. W. Miller, A. Moralez, J. Morales, M. Mutterer, A. Nikolaidis, A. Ortiz, T. Papaevangelou, A. Placci, G. Raffelt, J. Ruz, H. Riege, M. Sarsa, I. Savvidis, W. Serber, Y. Semertzidis, L. Stewart, J. Vieira, J. Villar, L. Walckiers, and K. Zachariadou, "First results form the cern axion solar telescope (cast)," Phys. Rev. Lett. 94, pp. 121301-1, 2005.

2. S. Weinberg, "A new light boson ?," Phys. Rev. Lett. 40, pp. 223-226, Jan. 1978.

3. F. Wilczek, "Problem of strong p and t invariance in the presence of instantons," Phys. Rev. Lett. 40, pp. 279-282, Jan. 1978.

4. R. D. Peccei and H. R. Quinn, "CP conservation in the presence of pseudoparticles," Phys. Rev. Lett. 38, pp. 14401443, June 1977.

5. G. G. Raffelt, "Axions: Recent searches and new limits," in Proc. XI International Workshop on Neutrino Telescopes,, (Venice, Italy), Apr. 2005. arXiv:hep-ph/0504152.

6. P. Sikivie, "Experimental tests of the invisible axion," Phys. Rev. Lett. 51, pp. 1415-1417, Oct. 1983.

7. K. van Bibber, P. M. McIntyre, D. E. Morris, and G. G. Raffelt, "Design for a practical laboratory detector for solar axions," Phys. Rev. D 39, pp. 2089-2099, Apr. 1989.

8. J. Altmann, W. J. Egle, U. Bingel, W. Hafner, B. Gaenswein, H. Schwarz, and A. Neugschwender, "Mirror system for the German X-ray satellite ABRIXAS: I. Flight mirror fabrication, integration, and testing," in Hoover and Walker. ${ }^{17}$

9. W. J. Egle, J. Altmann, P. Kaufmann, H. Muenker, G. Derst, H. Schwarz, and A. Neugschwender, "Mirror system for the German X-ray satellite ABRIXAS: II. Design and mirror development," in Hoover and Walker. ${ }^{17}$

10. M. Kuster, H. Bräuninger, J. Englhauser, J. Franz, P. Friedrich, R. Hartmann, D. Kang, R. Kotthaus, G. Lutz, W. Serber, and L. Strüder, "The x-ray mirror telescope and the pn-ccd detector of cast," in Gravitational Wave and Particle Astrophysics Detectors, J. Hough and G. H. Sanders, eds., Proceedings of SPIE 5500, SPIE, (Bellingham, WA), 2004.

11. G. Lutz, H. Bräuninger, J. Englhauser, R. Hartmann, D. Kang, R. Kotthaus, M. Kuster, W. Serber, and L. Strüder, "An application of space technology to the terrestrial search for axions: The X-ray mirror telescope at CAST," Nuclear Instruments and Methods in Physics Research A 518, pp. 201-206, Feb. 2004. 
12. L. Strüder, U. Briel, K. Dennerl, R. Hartmann, E. Kendziorra, N. Meidinger, E. Pfeffermann, C. Reppin, B. Aschenbach, W. Bornemann, H. Bräuninger, W. Burkert, M. Elender, M. Freyberg, F. Haberl, G. Hartner, F. Heuschmann, H. Hippmann, E. Kastelic, S. Kemmer, G. Kettenring, W. Kink, N. Krause, S. Müller, A. Oppitz, W. Pietsch, M. Popp, P. Predehl, A. Read, K. H. Stephan, D. Stötter, J. Trümper, P. Holl, J. Kemmer, H. Soltau, R. Stötter, U. Weber, U. Weichert, C. von Zanthier, D. Carathanassis, G. Lutz, R. H. Richter, P. Solc, H. Böttcher, M. Kuster, R. Staubert, A. Abbey, A. Holland, M. Turner, M. Balasini, G. F. Bignami, N. La Palombara, G. Villa, W. Buttler, F. Gianini, R. Lainé, D. Lumb, and P. Dhez, "The European Photon Imaging Camera on XMM-Newton: The pn-ccd camera," Astron. Astrophys. 365, pp. L18-L26, Jan. 2001.

13. M. Popp, R. Hartmann, H. Soltau, L. Strüder, N. Meidinger, P. Holl, N. Krause, and C. von Zanthier, "Modeling the energy response of pn-CCDs in the 0.2-10 kev band," Nuclear Instruments and Methods in Physics Research A 439, pp. 567-574, Jan. 2000.

14. F. Haberl, U. G. Briel, K. Dennerl, and V. Zavlin, "Spectral response of the epic-pn detector: Basic dependences," in New Visions of the X-ray Universe in the XMM-Newton and Chandra Era, F. Jansen, ed., ESA SP 488, ESA Publications Division, (Noordwijk), 2002.

15. G. Heusser, "Low-radioactivity background techniques," Annu. Rev. Nucl. Part. Sci. 45, p. 543, 1995.

16. J. F. Ziegler, "Terrestrial cosmic ray intensities," IBM Journal of Research and Development 42, pp. 117-139, Jan. 1998.

17. R. B. Hoover and A. B. Walker, eds., X-Ray Optics, Instruments, and Missions, Proceedings of SPIE 3444, (Bellingham, WA), SPIE, 1998. 